\title{
Análisis de las políticas culturales en el Perú
}

Analysis of cultural policies in Peru

(D) Fernando Pino Apablaza

Universidad Le Cordon Bleu. Lima, Perú

Recibido: 30/01/2021

Revisado: $18 / 04 / 2021$

Aceptado: 08/05/2021

Publicado: 31/ 06 / 2021

\section{RESUMEN}

La investigación se realizó en la ciudad de Lima, en el primer trimestre del año 2021. Tuvo como objetivos: conceptualizar la política, la cultura, y las políticas culturales. Además, identificar las principales problemáticas respecto a políticas culturales en la administración gubernamental, describir las leyes peruanas que atañen a la cultura, proponer la cultura como factor de desarrollo, y finalmente describir una taxonomía de las industrias culturales y creativas. Los objetivos de investigación, se cumplieron a través del análisis documental. Finalmente, la conclusión decanta en que las políticas culturales públicas en el Perú, son débiles e incipientes. Y para corolario las autoridades de turno no le prestan la debida atención e importancia, considerando a la cuestión cultural como algo secundario en el desarrollo de la comunidad. Palabras clave: Política, cultura, políticas culturales, industrias culturales, industrias creativas.

\begin{abstract}
The investigation was donde in Lima city, in the first trimester of 2021. Had like objectives: conceptualize the policy, the culture, and cultural policies. Also, identify the main problematics about the cultural policies in the governmental administration, describe the peruvian laws about the culture, propose the culture like a development factor and finally, describe a taxonomy about the cultural and creatives industries. The objectives of investigation were done, through the documental analysis. Finally the conclusion was that the cultural public policies are incipient and weaks. And the authorities on duty, dont put the right atention and importance, considering the cultural points like something in second stage in the community development.
\end{abstract} Keywords: Policy, culture, cultural policies, cultural industries, creatives industries. 


\section{INTRODUCCIÓN}

Para Nivón (2017), profesor investigador del Departamento de Antropología de la Universidad Autónoma Metropolitana de Iztapalapa en México, la demanda de políticas culturales eficaces ha incrementado. Siendo los primeros en mostrar interés los artistas e intelectuales, luego han estado los gestores o activistas de las áreas culturales de diversas instituciones, y ahora en el presente son los mismos ciudadanos los que demandan de políticas culturales eficaces e inclusivas. Y es natural, ya que el acceso a la cultura es para todos. Esta idea tiene asidero en el artículo $27^{\circ}$ de la Declaración Universal de los Derechos Humanos, que dicta que toda persona tiene derecho a tomar parte libremente en la vida cultural de la comunidad, a gozar de las artes y participar en el progreso científico y en los beneficios que de él resulten. En este mismo sentido Yúdice (2019) afirma que "gran parte de las políticas culturales que se vienen estableciendo en el nuevo milenio, tienen como marco los derechos culturales" (p.2).

\section{¿Qué es la política?}

Desde el análisis etimológico de la palabra política, se sabe que esta, tiene su origen en el vocablo griego Politeia, que significa teoría de la ciudad. Otro término relacionado a la política también de origen griego es politiké techne, que vendría a ser arte social o arte de vivir en sociedad, ideal que fue postulado por Aristóteles. Un antecedente predecesor al de Aristóteles, fueron obras literarias como La República y posteriormente Las Leyes, ambas obras de autoría de Platón.

Según Bracamonte (2002), "la política debe entenderse como un proceso en virtud del cual los hombres y grupos toman decisiones para hacer frente a situaciones diversas" (p.74). El concepto de política está asociado al concepto de Estado, siguiendo a Bracamonte "es la institución a través de la cual las acciones políticas se organizan en función del bien común, constituyéndose en una red de relaciones sistemáticas regidas por la ley” (p.76).

Para Roiz, como se citó en Guzmán (2008), respecto a la política esta es:

Un fenómeno universal en el que la persona humana desarrolla actividades y establece relaciones que responden a diferenciados sentidos y ámbitos de aplicación. La política está ligada a la misma condición humana y por lo tanto, puede considerarse como una construcción mental (p.6).

A raíz de las discusiones y consensos políticos se generan las leyes que son reglas que se disponen con un ideal, que es el bien común en la sociedad. Un ejemplo de las primigenias leyes en el mundo, ocurrió en el siglo XVIII antes de Cristo, en tiempos del imperio babilónico, en Mesopotamia, donde existieron los códigos del rey Hamurabi, que eran una serie de leyes basadas en la famosa ley del Talión, del ojo por ojo, diente por diente. Estos códigos fueron creados desde el poder del imperio, y como es natural fueron creados para impartir justicia en la sociedad.

En Perú, las leyes de origen estatal nacen de poderes constituidos, que están debidamente alineados a La Constitución Política del Perú, que nace de un poder constituyente. Y estas leyes naturalmente tienen carácter de obligatoriedad. Sin embargo, existen normas creadas por organismos privados que son voluntarias, por ejemplo, las normas que crea la Organización Internacional de Estandarización (ISO), que 
son normas de estandarización, para mejorar la efectividad de los procesos en las empresas. Estas normas no son obligatorias, por lo tanto, no tienen fuerza jurídica y el empresariado las adopta por conveniencia y libre albedrío.

Leyes que tienen diferentes rangos y nombres. Por ejemplo, decreto supremo, decreto legislativo, ordenanzas municipales y normas legales. Normas que valga la redundancia, normalizan la conducta de los sujetos, guían su actuación. Entonces, como dijo el filósofo francés Michel Foucault, los sujetos están sujetos a ellas.

Pero en la reflexión de hoy en día se pueden distinguir dos corrientes de pensamientos sobre la política, así como afirma Del Águila, como se citó en Guzmán (2008) que:

Hay dos grandes grupos de concepciones sobre la política, en primer lugar, la política en sentido cooperativo $y$, en segundo lugar, la política en sentido conflictivo. En el primer caso, la política sería la actividad a través de la cual los grupos humanos toman decisiones colectivas. En el segundo, inspirada en la concepción maquiavélica, se entiende la política como una actividad de conflicto entre personas, grupos de interés, o visiones del mundo (p.273).

Por supuesto que la más conveniente de adoptar es la primera, que va en un sentido cooperativo. Sin embargo, en la realidad de la mayoría de países la política siempre traerá consigo debates, peleas, protestas y hasta muertes.

\section{¿Qué es cultura?}

La palabra cultura al ser polisémica, cuenta con gran variedad de acepciones. Según Cornejo (1987) “los antropólogos Kroeber y Kluc- khon recogieron más de doscientas definiciones de cultura. Y desde entonces a nuestros días el número se ha incrementado" (p.3). En este mismo sentido el filósofo ruso V. Mezhúiev afirma respecto a la cultura, que ningún otro fenómeno de las ciencias suscita tanta divergencia de puntos de vista, juicios y definiciones. Por lo tanto, es necesario hacer un deslinde terminológico sobre el concepto cultura.

Empecemos con una definición de cultura, desde la visión antropológica. Sebastián Salazar Bondy, como se citó en Jacinto (1997), define "la palabra cultura en el sentido neutral de la antropología, como el nombre de un sistema de valores, símbolos y actitudes con que un grupo humano responde a las solicitaciones y conflictos que provienen del mundo y la existencia" (p.113). Otra definición interesante de cultura, pero con un enfoque más expresivo es la definición de Pulido et al., como se citó en Pino (2018), que la refiere como:

La suma de prácticas o expresiones que el ser humano ha desarrollado en su proceso de adaptación y creación de su medio vital, expresiones como el lenguaje, el arte, el derecho, la historia, la política etc. Mientras que el enfoque interpretativo de la cultura es la capacidad que tienen las diferentes sociedades de dotar significado o valor a las prácticas o expresiones antes mencionadas (p.252).

La cultura también se define como todo lo que la gente hace, tiene, piensa, y como se comportan, pero en el interior de un grupo

humano, es decir una serie de códigos que se comparten, una serie de símbolos, una serie de red de significados que hacen que la vida sea manejable en sociedad (Huerta, 2012). 
Además, Huerta deconstruye la cultura y observa que existen tres grupos alternos, donde la primera idea de cultura que es la más común y cotidiana es la idea de una cultura de élite, donde se encuentra la música clásica, la ópera, el teatro. Menciona que también hay otro tipo de cultura denominada cultura tradicional, donde el ejemplo sería aquella idea congelada en el tiempo de indigenismo romántico, por ejemplo, la idea clásica de Macchu Picchu como un mundo andino congelado en el tiempo, homogéneo, tierno, pero ficticio. Y finalmente, está la cultura popular en contra posición a la cultura de élite, la cultura popular es la más consumida, es aquella cultura que se difunde masivamente en los medios televisivos, es una cultura que no pertenece a un grupo sofisticadamente poderoso, es aquella cultura no reconocida como tal, que también es llamada cultura chicha. En este mismo sentido Montiel (2004) afirma que "la cultura es uno de los elementos fundamentales para la constitución de la identidad de un pueblo" (p.49).

Mientras que para Sommer, profesora de la universidad de Harvard, la cultura ya no es una cosa de elite, sino que es para todos, también refiere que existen diferencias entre las definiciones de cultura, por ejemplo en la visión antropológica de cultura se tiende a respetar las prácticas que ya existen, mientras que el artista o humanista busca las prácticas que están por crearse, es decir, si uno toma una línea antropológica uno respeta paradigmas, mientras que en la línea del artista este rompe todos los paradigmas (Sommer, 2017).

Para Geertz, como se citó en Eufracio (2017), la cultura es "como un mecanismo regulador y homogéneo que se sitúa por fuera de los individuos para gobernar su conducta" (p.104).
Otra definición interesante y en un sentido divergente a las anteriores es la de Basail (2005) que afirma:

La cultura como recurso cobra legitimidad entre los políticos que deciden la inversión y protección cultural por su valor utilitario para disminuir los conflictos, procurar justicia social e impulsar el desarrollo económico; para, de esta manera, fortalecer a la sociedad civil como soporte del desarrollo del capital, promover el desarrollo del turismo, de las industrias culturales en general y de todas las que dependen de la propiedad intelectual (p.78).

Para la Organización de las Naciones Unidas para la Educación, Ciencia y Cultura (UNES$\mathrm{CO}$ ), la cultura es el complejo de características espirituales, materiales, intelectuales y emocionales distintivas de una sociedad o grupo social. Incluye, no solo las artes y las letras, sino también las formas de vida, los derechos fundamentales del ser humano, los sistemas de valores, las tradiciones y las creencias.

Para terminar este apartado sobre la cultura donde hemos podido observar una vasta variedad de conceptos, concluimos con la definición de Emile Durkheim como se citó en Urbano (2004), que la define sucintamente como "conciencia colectiva" (p.12).

\section{¿Qué son políticas culturales?}

Según Cortés (2006), en 1968 la UNESCO definió a las políticas culturales como:

Un conjunto de prácticas sociales, conscientes y deliberadas, de intervención o no intervención, que tienen por objeto satisfacer ciertas 
necesidades culturales de la población y de la comunidad, mediante el empleo óptimo de todos los recursos materiales y humanos que dispone una sociedad en un momento determinado (p.19).

Si bien las políticas culturales desde tiempos pretéritos se han desarrollado en un sentido proteccionista, hay que decir que, en el presente las políticas se han desarrollado en un sentido de difusión de la cultura. Así lo afirma Altuve (2000) "en los últimos años se ha introducido de manera enérgica el valor del concepto promoción de la cultura, que sirve como equilibrio ante la sola actividad protectora del Estado" (p.550).

Otra definición sobre las políticas culturales es la de Texeira (2009), que afirma lo siguiente:

La política cultural constituye una ciencia de la organización de las estructuras culturales y generalmente es entendida como un programa de intervenciones realizadas por el estado, instituciones civiles, entidades privadas o grupos comunitarios con el objeto de satisfacer las necesidades culturales de la población y promover el desarrollo de sus representaciones simbólicas (p.241).

En la definición anterior, se incorpora la responsabilidad de las instituciones privadas respecto a la intervención en política cultural. Otra definición muy similar es la de Néstor García Canclini, como se citó en Atehortúa (2008), que afirma que:

Las políticas culturales son el conjunto de intervenciones realizadas por diversos agentes. Tales como el Estado, organizaciones no gubernamentales o grupos comunitarios, con el objetivo de orientar el desarrollo simbólico y responder a las necesidades culturales de la población (p.127).

Otro concepto de política cultural es el de Gilberto Giménez, como se citó en Figueroa (2006), que afirma:

La política cultural surge precisamente de la intervención del estado y de los poderes públicos en el orden de la cultura. El Estado tiene buenas razones para interesarse por la cultura. Por ejemplo, a sus ojos la cultura funciona como fuente de legitimación, como fundamento y clave de la identidad nacional, como título de prestigio en la competencia internacional, como fuente de recursos en la economía nacional.

En el documento titulado Políticas Culturales 2017-2022 elaborado por el Consejo Nacional de la Cultura y las Artes del Gobierno de Chile, definen a las políticas culturales como un conjunto de orientaciones y decisiones que el Estado con la participación de organizaciones de la sociedad civil y grupos comunitarios, diseña y ejecuta con la finalidad de facilitar la consecución de objetivos considerados necesarios o deseables en el ámbito de la cultura en general o respecto de un sector cultural o disciplina específica, y sirve como cartas de navegación para la acción del Estado en el campo cultural, permitiendo definir lineamientos, prioridades y articular distintos ministerios, servicios y agentes culturales, para lograr que su accionar sea integral y efectivo. Leyes y políticas culturales en el Perú

En este apartado se describen las leyes nacionales que tratan sobre los derechos fundamentales del hombre respecto a la cultura, y además, sobre el rol que tiene el Estado. 
Artículo 2, inciso 8 de la Constitución Política del Perú (1993), que se encuentra en el capítulo titulado Derechos Fundamentales de la Persona, dicta lo siguiente:

Toda persona tiene derecho a la libertad de creación intelectual, artística, técnica y científica, así como a la propiedad sobre dichas creaciones y a su producto. El estado propicia el acceso a la cultura y fomenta su desarrollo y creación.

Cabe mencionar que el artículo $2^{\circ}$, inciso 8 de La Constitución Política del Perú está alineado a los Artículos $45^{\circ}$ y $50^{\circ}$, del Pacto de San José de Costa Rica (1969), ratificados en la Convención Americana sobre Derechos Humanos.

Artículo 45: Los Estados Miembros darán importancia primordial, dentro de sus planes de desarrollo, al estímulo de la educación, la ciencia y la cultura, orientadas hacia el mejoramiento integral de la persona humana y como fundamento de la democracia, la justicia social y el progreso (s/n).

Artículo 50: Los Estados Miembros acuerdan promover, dentro del respeto debido a la personalidad de cada uno de ellos, el intercambio cultural como medio eficaz para consolidar la comprensión interamericana y reconocen que los programas de integración regional deben fortalecerse con una estrecha vinculación en los campos de la educación, la ciencia y la cultura $(\mathrm{s} / \mathrm{n})$.

Además, existe la Ley General de Patrimonio Cultural de la Nación. Ley N²8296 (2004), que en su capítulo segundo, titulado Participación de Entidades Estatales, artículo 29 sobre Municipalidades, expresa lo siguiente:
Las competencias y funciones establecidas en la Ley Orgánica de Municipalidades, corresponde a las municipalidades en sus respectivas jurisdicciones:

a) Cooperar con el Instituto Nacional de Cultura, la Biblioteca Nacional y el Archivo General de la Nación en la identificación, inventario, registro, investigación, protección, conservación, difusión y promoción de los bienes muebles e inmuebles integrantes del Patrimonio Cultural de la Nación.

b) Dictar las medidas administrativas necesarias para la protección, conservación y difusión de los bienes integrantes del Patrimonio Cultural de la Nación de su localidad, en concordancia con la legislación sobre la materia y las disposiciones que dicten los organismos competentes.

c) Elaborar planes y programas orientados a la protección, conservación y difusión de los bienes integrantes del Patrimonio Cultural de la Nación de su localidad, en coordinación con los organismos competentes.

Las ordenanzas, resoluciones, acuerdos y reglamentos emitidos por las municipalidades que se refieran a bienes integrantes del Patrimonio Cultural de la Nación requieren opinión previa del organismo competente, en caso contrario serán nulas de pleno derecho $(\mathrm{s} / \mathrm{n})$.

También existe la Ley Orgánica de Municipalidades. Ley 27972 (2003), en su artículo $82^{\circ}$ sobre Educación, Cultura, Deportes y Recreación, expresa lo siguiente: 
Las municipalidades, en materia de educación, cultura, deportes y recreación, tienen como competencias y funciones específicas compartidas con el gobierno nacional y el regional entre otras la siguiente: promover la protección y difusión del patrimonio cultural de la nación, dentro de su jurisdicción, la defensa y conservación de los monumentos arqueológicos, históricos $\mathrm{y}$ artísticos, colaborando con los organismos regionales y nacionales competentes para su identificación, registro, control, conservación y restauración ( $\mathrm{s} / \mathrm{n})$.

\section{La cultura como factor de desarrollo}

La representante de UNESCO en Perú, afirma que "es importante que la cultura sea vista como un eje de desarrollo económico inclusivo. La cultura genera empleo, trabajo y obviamente en el caso de Perú hay un activo importantísimo". Además, la Jefa de la Oficina de UNESCO en Lima, señaló que sólo en el sector turismo, más del 70\% pertenece al turismo cultural. "Con una política enfocada de desarrollo económico, desde cultura y turismo, se puede activar la economía local y mejorar las condiciones de vida" (Robalino, s.f.)

Para el Estado, la cultura siempre ha desarrollado un papel sustantivo en la sociedad, al igual que la salud o la seguridad, si bien de los sectores antes mencionados, no se esperan retribuciones económicas, naturalmente por ser servicios públicos, se sabe con respecto a la cultura que más allá de generar identidad, ahora es considerada un factor que incide en el desarrollo de las naciones, tanto así que el Banco Interamericano de Desarrollo (BID), ha denominado a las industrias culturales y creativas como la economía naranja.
Ahora habría que hacerse la pregunta ¿Qué quiere decir economía naranja? Según Finlev, et al. (2017), esta se refiere a la "economía creativa y cultural" ( $\mathrm{s} / \mathrm{n})$, como abreviación del ecosistema definido por el BID en su informe La economía naranja. Abarca la economía creativa y cultural y sus industrias asociadas, sus actividades de apoyo creativo, el diseño, los nuevos medios de comunicación, el software, las artes y el patrimonio. En esta economía, el talento y la creatividad constituyen los principales insumos y recursos.

Esta economía naranja ha generado empleo a una gran cantidad de individuos involucrados en las diferentes actividades antes mencionadas. Según Helga Flores, Directora de Industrias Culturales del BID, el sector cultura, genera en el mundo 2300 millones de dólares al año, lo que es equiparable con el PBI de la India, que es el segundo país más grande en población en el planeta. Otro dato importante es que las industrias culturales y creativas en América Latina generan 11 millones de empleos. Además, se sabe que las industrias culturales aportan en el Perú el 2.7\% del PBI, superando la cifra del año 2007 que fue de 1.2\%.

Por lo tanto, las políticas culturales más allá de ir en un sentido proteccionista y difusor del patrimonio, como se ha podido observar en el apartado anterior, titulado Políticas culturales en el Perú, estas deberían ir más bien en un sentido de facilitación y apoyo con respecto a los actores y agentes de las industrias culturales y creativas. Según Grimaldo (2006) "en algunos países desarrollados la política cultural forma parte del progreso político, económico y social que ha alcanzado el Estado, asumiéndola como uno de los componentes más importantes, a partir de la cual se hace viable el desarrollo" (p.46). 


\section{Problemáticas de la política cultural a nivel local}

Los problemas son situaciones que se deben de resolver, y en el campo de las políticas culturales, como dijo Cesar Vallejo "hay mucho por hacer". Según Yanovich (2017), representante de la Alianza Peruana de Organizaciones Culturales (APOC), mencionó en el último Foro Internacional de Cultura, realizado por el MINCUL, que:

\section{La APOC tiene como objetivos fortalecer} e impulsar la articulación de las organizaciones culturales, incidir en el diseño, implementación y evaluación de políticas culturales reconociendo la transversalidad de la cultura, y propiciar e institucionalizar espacios y mecanismos que garanticen el dialogo y colaboración entre el Estado y sociedad civil ( $\mathrm{s} / \mathrm{n})$.

Además, Yanovich (2017) identificó las principales problemáticas que se observan en los gobiernos locales y que se consideran brechas que inciden en el desarrollo de una gestión eficaz de cultura. A continuación, se describen los problemas identificados:

Problema 1. La visión de cultura como factor de desarrollo no es compartida por todos los actores y sectores de la sociedad. Las autoridades desconocen el rol de la cultura como factor que mejora la calidad de vida de los ciudadanos.

Problema 2. La propia ciudadanía tiende a subestimar el papel que juega la cultura en la generación de comunidades más sostenibles, saludables, seguras, inclusivas y democráticas.
Problema 3. La ausencia de políticas culturales, y si hay no se cumplen. Esto pone en riesgo la sostenibilidad de esfuerzos para poner en marcha proyectos culturales, y limita las oportunidades entre Estado y sociedad civil. Además, los esfuerzos por generar políticas culturales intersectoriales que abordan los vínculos del sector cultural con salud, educación, seguridad, medio ambiente se ven seriamente limitados.

Problema 4. La falta de apoyo de las autoridades locales y regionales en las dinámicas de transformación social desde la cultura.

Problema 5. Falta de transparencia y medición de impacto, no se cuenta con instrumentos que midan el impacto de las políticas culturales, no se cuenta con información suficiente de iniciativas y programas culturales impulsados por el Estado ( $/ \mathrm{n}$ ).

Como se puede observar, las problemáticas planteadas en párrafos anteriores, respecto a las políticas culturales. Atañen directamente a las autoridades en sus diferentes niveles; ya sean regionales, provinciales, distritales. Son las autoridades las que deben poner esfuerzos en impulsar la gestión cultural al servicio del público. Según Arroyo y Carmona, como se citó Cristian (2011), respecto a la evaluación de las políticas públicas afirma que:

En la evaluación de las políticas públicas incluirá también al conjunto de decisiones que los Estados toman en el amplio campo del arte y la cultura, especialmente desde que el 
acceso y el disfrute de las mismas aparecieron consignadas como un derecho humano esencial en la Declaración Universal (p.52) Un derecho que no se ve manifiesto, ya que la cultura no llega a todos. La idea de democratizar la cultura, consigna un verdadero desafío que deberá ser asumido con la mayor equidad posible, procurando acercar la cultura y todas sus expresiones a la mayor cantidad de personas posibles. En este mismo sentido Espinoza et al. (2016) refieren que "la implementación de las políticas culturales está íntimamente relacionadas con la difusión y promoción de las artes como herramienta para la creación de una identidad y cohesión social, así como con la intención de impulsar y mejorar los procesos educativos" (p.5). Entonces, si la cultura trae consigo: identidad, cohesión y educación; habría que reflexionar por qué las autoridades no muestran interés en la gestión cultural, ni en el fortalecimiento de las políticas públicas.

\section{Las dimensiones de las industrias cultura- les y creativas}

En este apartado revisaremos las dimensiones, ámbitos o sectores que conforman las industrias culturales y creativas, desde la mirada de expertos. Según Towse (2017), profesora de Economía de Industrias Culturales de la Universidad de Bournemouth en el Reino Unido, menciona sobre las industrias creativas, que:

Son una mezcla entre la cultura de élite y de la cultura popular, y que ambas tienen diferentes perfiles económicos. Los objetivos de las políticas en las industrias creativas son incrementar los servicios de cultura, pero también aumentar su alcance en la comunidad. Los ámbitos que figuran en la lista del Departamento de Medios Digitales Culturales y Deportes del Reino Unido son la publicidad y marketing, arquitectura, artesanía, diseño, cine, televisión y radio, tecnologías de información, servicios de software y computación, museos, galerías y librerías, música, artes visuales y publicaciones $(\mathrm{s} / \mathrm{n})$.

Para Flores (2017), directora del equipo de Industrias Culturales del Banco Interamericano de Desarrollo, las industrias creativas y culturales son:

Parte de la economía naranja, que son aquellos bienes y servicios basados en la propiedad intelectual que nacen del talento, del patrimonio de la creatividad. La economía naranja se refiere entonces a todos los sectores económicos que están en el entre cruce entre cultura, arte, tecnología y comercio y abarca desde las artes tradicionales, las industrias creativas y los servicios anexos de soporte es decir el cine, la moda, video juegos y el diseño (s/n).

Mientras que para la UNESCO (2010), según su Guía para el desarrollo de las industrias culturales y creativas son:

Los sectores que están circunscritos a las actividades de las industrias culturales y creativas se dividen en seis ámbitos que son el patrimonio natural y cultural que incluyen los productos derivados y servicios de los museos, sitios arqueológicos, históricos y paisajes culturales; las presentaciones artísticas y celebraciones donde se incluyen las artes escénicas, la música, festivales, festividades y ferias; los medios audiovisuales y creativos que incluyen filmes, videos, radio, televisión, podcasting, mainstreaming, juegos de video y de animación; 
los diseños y servicios creativos que incluyen la moda, el diseño gráfico, diseño de interior, paisajismo, servicios de arquitectura y de publicidad; los libros y prensa que incluyen libros, periódicos, otros materiales impresos, bibliotecas $\mathrm{y}$ ferias de libros; las artes visuales $\mathrm{y}$ artesanía que incluyen la pintura, la escultura, la fotografía y la artesanía ( $\mathrm{s} / \mathrm{n}$ ). Referente a las anteriores, es la del profesor de la Universidad de Miami, George Yúdice, que junto a Sylvie Duran, fabricaron un cuadro sinóptico donde se reflejan los ámbitos de la economía creativa.

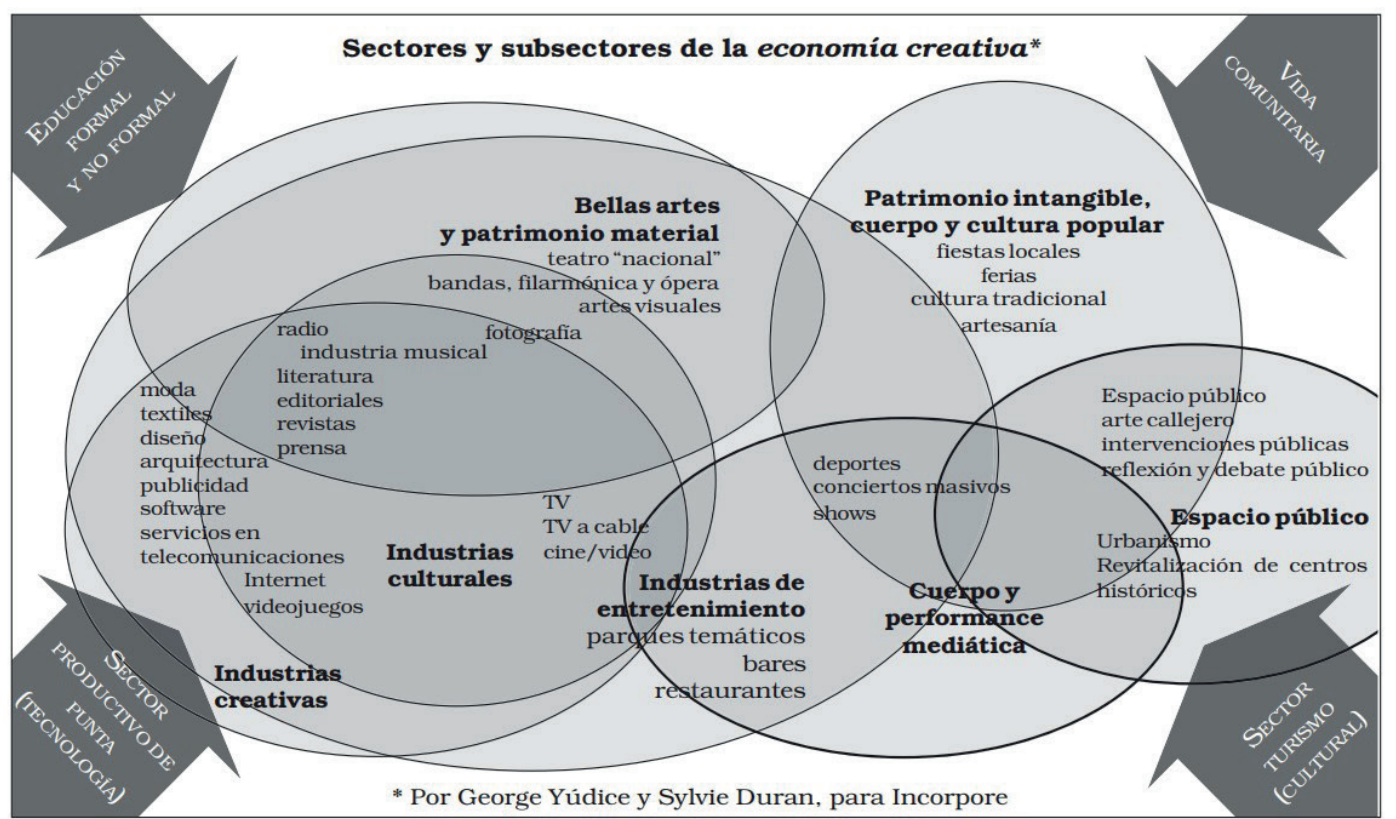

Elaborado por: Yúdice y Duran, 2018.

Figura 1. Sectores y subsectores de la economía creativa

\section{CONCLUSIONES}

El concepto de cultura al ser polisémico. Tiene diferentes acepciones, dependiendo el contexto en el que se encuentre. Pero dentro de este contexto político, la acepción pertinente sería aquella que va en un sentido antropológico de cultura viva donde el interés está en las costumbres, los hábitos, las formas de expresión y creación. Al fin y al cabo, la cultura es creación humana. Las políticas culturales públicas son débiles e incipientes. Los problemas que giran en torno a la cultura son secundarios para las autoridades de turno. Esta situación es común, sobre todo en países del tercer mundo, don Otra clasificación muy parecida pero di de los ineficientes gobernantes no sopesan la importancia que tiene la cultura como eje de desarrollo. Sin embargo, en otras latitudes se observa la situación inversa, donde se manifiesta verdadera preocupación desde las universidades y gobernanzas por seguir impulsando a la cultura como un objetivo primordial a nivel país y que, además, aporta réditos significativos. Lo paradójico de todo esto, es que el Perú es considerado como un país multicultural, que podría aprovechar esta ventaja para mostrarse al mundo, ofertando un amplio abanico de posibilidades. Pero en gran medida todo esto dependerá de las políticas públicas y las autoridades de turno. 


\section{REFERENCIAS BIBLIOGRÁFICAS}

Altuve Febres, F. (2000). Patrimonio Cultural del Perú. Lima: Fondo Editorial del Congreso del Perú.

Atehortúa Castro, L. (2008). Políticas públicas de cultura: un rasgo de la relación entre miedos y esperanzas, el caso del municipio de Bello, 1997-2007. Estudios Políticos(33), 123-145. doi:ISSN 0121-5167

Basail Rodríguez, A. (2005). Desarrollo y políticas culturales. Adagio al discurso y al recurso de la cultura. LiminaR, 3(1), 74-99. doi: https://dx.doi.org/10.29043/liminar.v3i1.170

Bracamonte E, E. (2002). Política, Estado y gobierno. Ciencia y Cultura (10), 73-78. ISSN 2077-3323

Constitución Política del Perú. (31 de diciembre de 1993). Derechos Fundamentales de la Persona. Lima, Perú. http:// www.congreso.gob.pe/Docs/files/constitucion/constitucion-politica-14-03-18.pdf

Cornejo Polar, J. (1987). Cuadernos de historia. Lima: Universidad de Lima.

Cortés, G. (2006). Politicas culturales. Lima.

Cristian , A. (2011). Control y evaluación de las políticas culturales en Chile. Universum, 1(26), 13-37. doi: http://dx.doi.org/10.4067/ S0718-23762011000100002

Eufracio Jaramillo, J. (2017). La Cultura y la política en la cultura política. Nueva antropología, 30(86), 101-119. doi:ISSN 0185-0636

Figueroa Díaz, M. (2006). Políticas culturales para el desarrollo en un contexto mundializado. Política y cultura(26), 157-183.
Recuperado el 29 de marzo de 2020, de http://www.scielo.org.mx/scielo.php?script $=$ sci_arttext\&pid=S0188-77422006000200008

Finlev, T., Maguire, R., Oppenheim, B., \& Skvirsky, S. (2017). El futuro de la economía naranja. Formulas creativas para mejorar vidas en América Latina y el Caribe. Palo Alto: BID.

Flores, H. (27 de octubre de 2017). Ministerio de Cultura Perú. Foro Internacional de Cultura: https://www.youtube.com/watch?v=_tuIQ3jHU_M\&t=56s

Grimaldo Muchotrigo, M. (2006). Identidad y política en el Perú. Liberabit(12), 41-48. http://www.scielo.org.pe/pdf/liber/v12n12/ a03v12n12.pdf

Guzmán Mendoza, C. (2008). La política como objeto de reflexión. Revista de Derecho(30), 268-298. doi:ISSN 0121-8697

Guzmán Mendoza, C. E. (2008). La política como objeto de reflexión. Revista de Derecho(30), 268-298. http://www.scielo.org.co/scielo.php?script=sci_arttext\&pid=S0121-86972008000200010

Huerta, A. (29 de febrero de 2012). PUCP. ¿Qué es la cultura?: ht t p s://ww w.youtube.com/wat $\mathrm{ch} ? \mathrm{v}=\mathrm{CLLVIFA} 6 \mathrm{~d} \mathrm{~J}$ M \& t $=183 \mathrm{~s}$ Jacinto Pazos, P. (1997). Notas sobre el concepto de cultura en el Perú. Lima: UNMSM.

Ley $\mathrm{N}^{\circ} 27972$. (27 de mayo de 2003). Ley Orgánica de Municipalidades. Lima, Perú.

https://www.mef.gob.pe/contenidos/presu_publ/capacita/programacion_formulacion_presupuestal2012/Anexos/ley27972.pdf 
Ley $\mathrm{N}^{\circ} 28296$. (22 de julio de 2004). Ley General del Patrimonio Cultural de la Nación. Participación de Entidades Estatales. Lima, Perú. https://www.peru.gob.pe/docs/ PLANES/94/PLAN_94_LEY\%20N\%C2\%BA\%2028296_2008.pdf

Montiel, E. (2004). La diversidad cultural en la era de la globalización. Turismo y $\mathrm{Pa}$ trimonio(4), 47-55. doi:ISSN: 1680-9556

Nivón, E. (26 de octubre de 2017). Ministerio de Cultura del Perú. Foro Internacional de Cultura: https://www.youtube.com/watch? $\mathrm{v}=\mathrm{k} 7 \mathrm{zG} 0 \mathrm{DdLf} 74 \& \mathrm{t}=330 \mathrm{~s}$

rtiz Espinoza, Á., Gutierrez Díaz, M., \& Hernández Alba. (2016). Identidad, cohesión y patrimonio: evolución de laspolíticas culturalesen México. Humanidades, 6(6), 1-39. https://www. redalyc.org/articulo.oa?id $=498054743002$

Pacto de San José de Costa Rica. (22 de noviembre de 1969). https://www.oas.org/ dil/esp/tratados_b-32_convencion_americana_sobre_derechos_humanos.htm

Pino Apablaza, F. (2018). La educación superior y el patrimonio cultural. Scientia, 20(18), 247-266. doi:ISSN1993-422X

Robalino, M. (s.f.). UNESCO en Perú: La cultura es un eje de desarrollo económico inclusivo. http://www.unesco.org/new/es/ media-services/single-view-tv-release/news/ unesco_en_peru_la_cultura_es_un_eje_de desarrollo_economi/
Sommer, D. (26 de octubre de 2017). Ministerio de Cultura Perú. Obtenido de https:// www.youtube.com/watch? $\mathrm{v}=\mathrm{k} 7 \mathrm{zG} 0 \operatorname{DdLf} 74$ Teixeira , C. (2009). Diccionario crítico de política cultural. Barcelona: Gedisa, S.A. Towse, R. (27 de octubre de 2017). Ministerio de Cultura Perú. Foro Internacional de la Cultura: https://www. youtube.com/watch?v=_tuIQ3jHU_M

UNESCO. (2010). Políticas para la creatividad: Guía para el desarrollo de las industrias culturales y creativas. París.

Urbano , H. (2004). La cultura como horizonte. Entre la tradición y la modernidad. Turismo y Patrimonio(4), 11-20. doi:ISSN: 1680-9556 Yanovich, Y. (26 de octubre de 2017). Ministerio de Cultura Perú. Foro Internacional de Cultura: https://www. youtube.com/watch?v=b9aY $165 \mathrm{giuQ}$

Yúdice, G. A. (2019). Políticas culturales y ciudadanía. Educacao \& Realidade, 44(4), 1-24. doi:https://doi.org/10.1590/2175-623689221 